\title{
TRPV1 Is Associated with Testicular Apoptosis in Mice
}

\author{
Adrian S. Siregar, ${ }^{1,2, \#}$, Marie Merci Nyiramana,2,\#, Eun-Jin Kim, Eui-Jung Shin ${ }^{1,2}$, \\ Chang-Woon Kim ${ }^{3}$, Dong Kun Lee ${ }^{1,2}$, Seong-Geun Hong ${ }^{1,2}$, Jaehee Han ${ }^{1}$ and Dawon Kang ${ }^{1,2, *}$ \\ ${ }^{1}$ Department of Physiology, College of Medicine and Institute of Health Sciences, Gyeongsang National University, Jinju \\ 52727, Korea \\ ${ }^{2}$ Department of Convergence Medical Science, Gyeongsang National University, Jinju 52727, Korea \\ ${ }^{3}$ Department of Obstetrics and Gynecology, Samsung Changwon Hospital, Sungkyunkwan University School of \\ Medicine, Changwon 51353, Korea
}

Received December 15, 2019

Revised December 27, 2019

Accepted December 27, 2019

\section{*Correspondence}

Dawon Kang

E-mail: dawon@gnu.ac.kr

ORCID

https://orcid.org/0000-0001-7402-7298

"These authors contributed equally to this work.

\begin{abstract}
Reproductive potential decreases with age. A decrease in male fertility is due to a combination of morphological and molecular alterations in the testes. Transient receptor potential vanilloid receptor-1 (TRPV1) is associated with aging and lifespan, and its activation causes apoptotic cell death in various cell types. However, the effect of TRPV1 on testicular apoptosis in aged mice has not yet been reported. TRPV1 knockout (KO) mice had a longer lifespan than that of wild-type (WT) mice. Lifespan was increased by $11.8 \%$ in male TRPV1 KO mice compared to that in WT mice. TRPV1 KO males lived approximately 100 days longer than WT males on average, and the maximum lifespan was markedly extended in TRPV1 KO mice compared with that in WT mice. The TRPV1 expression levels were highly increased in the testes of older mice. TRPV1 was expressed in the entire testes region of the old mice. In addition, old TRPV1 KO mice had lower testicular apoptosis than that of WT mice. Our results show that TRPV1 induces testicular apoptosis and suggest that TRPV1 may be associated with testicular aging.
\end{abstract}

Keywords: aging, apoptosis, mice, testes, TRPV1 receptor

\section{INTRODUCTION}

The decline of reproductive activity occurs in a way that is specific to species and gender. Reproductive activity in women ends upon menopause, whereas there is no complete cessation of reproductive capacity in men (Tenover, 2003). In men, a combination of morphological and molecular changes in reproductive organs with advanced age may lead to a decline in reproductive activity (Handelsman, 2006). Recent research has shown that mice lacking the transient receptor potential vanilloid receptor-1
(TRPV1) pain receptors have a long-lived and youthful metabolic profile at an older age (Riera et al., 2014).

TRPV1 is a nonselective cation channel that was originally found to be expressed in sensory neurons. TRPV1 is activated by heat, protons, capsaicin, and endovanilloids and functions as a multimodal detector of noxious stimuli (Caterina et al., 1997). TRPV1 knockout (KO) mice show impaired nociception and pain sensation (Caterina et al., 2000). Subsequent studies have shown that TRPV1 is expressed in non-neuronal cells, including male germ cells, and its activation is associated with cell protection 
and apoptosis (Mizrak et al., 2008; Mizrak and van DisselEmiliani, 2008; De Toni et al., 2016). TRPV1 KO mice have a testis phenotype that is much more vulnerable to cell death induced by oxidative stress stimuli and massive loss of germ cells from seminiferous tubules compared to wild-type (WT) mice (Mizrak and van Dissel-Emiliani, 2008; Martins et al., 2014).

Apoptosis has been found to play an important role in the aging process (Muradian et al., 2002). Apoptosis pathways are considered as key targets for delaying or reducing aging, because cell damage and inappropriate systemic signals accumulated during aging can lead to abnormal cell death and limit body function and lifespan (Shen and Tower, 2009). Although it has been found that the lifespan of TRPV1 KO mice is extended, the degree of aging, particularly in the testes, has not been addressed. Aging processes, regulated by genetic and environmental factors, can affect the lifespan. Programmed cell death that occurs during aging could also be associated with a shortened lifespan. This study was performed to investigate the relationship between TRPV1 expression and testicular apoptosis using 32-month-old WT and TRPV1 KO mice.

\section{MATERIALS AND METHODS}

\section{Chemicals}

All components of the medium and other chemicals were tissue culture grade and were purchased from Sigma Chemical Co. (St. Louis, MO, USA), unless otherwise specified.

\section{Animals and testis isolation}

WT and TRPV1 KO male mice (C57BL/6J, six-week-old) were obtained from Central Lab. Animal Inc. (Seoul, Korea) and professor Dong Kun Lee (Gyeongsang National University, dklee@gnu.ac.kr), respectively. The mice were housed in a pathogen-free area under a 12-h light/dark cycle, with free access to food and water. The testes were isolated from mice that died at 32 months of age. Animal experiments were performed in accordance with the guidelines of the Gyeongsang National University Animal Care and Use Committee (GNU-161219-M0072). Individuals were monitored daily and weighed monthly but were otherwise undisturbed until they died. Survival curves were built using known birth and death dates.

\section{Genotyping}

To identify transgenes, before the experiment, TRPV1 $\mathrm{KO}$ mice were genotyped according to the protocol provided by Jackson Laboratories (Bar Harbor, ME, USA). DNA from the tail was extracted using the Phire Tissue Direct PCR Master Mix (Thermo Fisher Scientific Baltics UAB, Vilnius, Lithuania), and the concentration was determined using a NanoDrop spectrophotometer (ND-1000, NanoDrop Technologies, Wilmington, USA). To detect TRPV1 gene truncation in TRPV1 KO mice, forward primers (Jackson Laboratory; \#19922, 5'-TGGCTCATATTTGCCTTCAG-3' for WT and \#OIMR1627, 5'-TAAAGCGCATGCTCCAGACT-3' for TRPV1 KO) and a common reverse primer (Jackson Laboratory, \#19923; 5' CAGCCCTAGGAGTTGATGGA 3') were used to produce gene products.

\section{Reverse transcriptase-polymerase chain reaction (RT-PCR)}

Total RNA was isolated from mice testes using TRIzol ${ }^{\mathrm{TM}}$ Reagent (Invitrigen, Carlsbad, CA, USA) according to the manufacturer's instructions. Briefly, the tissues were incubated in the isopropanol and TRIzol ${ }^{\mathrm{TM}}$ Reagent for lysis. The tissue lysate was precipitated by centrifugation for 10 $\min$ at $12,000 \times \mathrm{g}$ at $4^{\circ} \mathrm{C}$. The RNA pellets were washed in $75 \%$ ethanol, air-dried, and solubilized in RNase-free water. The isolated total TNA $(3 \mu \mathrm{g})$ was used to synthesize the first-strand cDNA using a reverse transcriptase kit (DiaStartTM RT kit; SolGent, Daejeon, Korea). PCR amplification was performed by using first-strand cDNA, Taq polymerase (G-Taq, Cosmo Genetech, Seoul, Korea), and specific primers for mouse TRPV1 (\#NM_001001445.2, forward: 5'-TCACCGTCAGCTCTGTTGTC-3' and reverse: AAGAAGTCCCCGTTAGCAGC). Glyceraldehydes3-phosphate dehydrogenase (GAPDH, \#NM_017008, forward: 5'-CTAAAGGGCATCCTGGGC-3' and reverse: 5' -TTACTCCTTGGAGGCCATG-3') was used as a loading control. The PCR conditions included initial denaturation at $94^{\circ} \mathrm{C}$ for $5 \mathrm{~min}$, then 32 cycles at $94^{\circ} \mathrm{C}$ for $30 \mathrm{~s}, 60^{\circ} \mathrm{C}$ for $30 \mathrm{~s}$, and $72^{\circ} \mathrm{C}$ for $30 \mathrm{~s}$, and a final extension step at $72^{\circ} \mathrm{C}$ for $10 \mathrm{~min}$. The PCR products were electrophoresed on a $1.5 \%(\mathrm{w} / \mathrm{v})$ agarose gel to verify the product size. The images of DNA fragments were directly captured with a gel imaging system using a digital camera (Canon, Tokyo, Japan) and an ultraviolet transilluminator (Vilber Lourmat, Marne La Vallee, France). The DNA fragments were directly sequenced with the ABI PRISM ${ }^{\circledR} 3100$-Avant Genetic 
Analyzer (Applied Biosystems, CA, USA).

\section{Hematoxylin and eosin (H\&E) staining}

Histological changes in testes were analyzed by staining with hematoxylin and eosin (H\&E) solution. The tissues were fixed in $4 \%$ paraformaldehyde solution overnight at $4^{\circ} \mathrm{C}$, washed in $0.1 \mathrm{M} \mathrm{PBS}$, embedded in paraffin, and cut into $5 \mu \mathrm{m}$-thick sections. The sections were air-dried on gelatin-coated slides, deparaffinized, and washed with tap water for $5 \mathrm{~min}$. After washing, the sections were immersed in hematoxylin solution for $5 \mathrm{~min}$. The degree of hematoxylin staining was verified in tap water, followed by eosin staining for $1 \mathrm{~min}$. The sections were dehydrated through a graded series of alcohols ( $70 \%$ to $100 \%$ ethanol, 3 min each), cleared in xylene, and mounted with permount mounting medium (Fisher Chemical, Geel, Belgium). The stained sections were observed and photographed using a BX61VS microscope (Olympus, Tokyo, Japan). Five sections from each sample were evaluated.

\section{Immunohistochemistry (IHC)}

To identify the expression and localization of TRPV1, the testis sections were processed by immunostaining. Deparaffinized tissue sections were permeabilized with $0.2 \%$ Triton $\mathrm{X}-100$ for $10 \mathrm{~min}$ at room temperature. After three washes in PBS, the sections were incubated with blocking buffer (10\% normal goat serum in $0.1 \mathrm{M}$ PBS) for $60 \mathrm{~min}$ at room temperature. Without washing, the sec- tions were then incubated with rabbit polyclonal antiTRPV1 (1:200 dilutions, Alomone Lab, Jerusalem, Israel) primary antibody overnight at $4^{\circ} \mathrm{C}$. After three washes in PBS, the sections were incubated in the dark for $1.5 \mathrm{~h}$ with FITC-conjugated anti-rabbit IgG secondary antibody (Abcam, Cambridge, UK) diluted at 1:400 in PBS. Finally, the sections were washed three times in PBS and stained with propidium iodide (PI) for nuclei staining. The stained sections were wet-mounted with Gel/Mount ${ }^{\mathrm{TM}}$ (Biomeda Corp., Foster City, CA, USA) and observed using a confocal laser scanning microscope (Olympus, Tokyo, Japan).

\section{TUNEL staining}

Apoptotic signal in the testes was assessed using the DeadEnd $^{\text {TM }}$ Fluorometric TUNEL System (Promega, Madison, WI, USA) according to the manufacturer's protocol. Briefly, paraffin-embedded testis sections were deparaffinized, washed, rehydrated, and washed again. The tissue slides were fixed in $4 \%$ paraformaldehyde in PBS for $15 \mathrm{~min}$ at room temperature, washed three times in PBS, and permeabilized with $20 \mu \mathrm{g} / \mathrm{mL}$ proteinase $\mathrm{K}$ solution for $10 \mathrm{~min}$ at room temperature. After three washes in PBS, the slides were refixed in $4 \%$ paraformaldehyde for 5 min at room temperature. The slides were washed in PBS for $5 \mathrm{~min}$ and equilibrated in an equilibration buffer for $10 \mathrm{~min}$. The tissues were labeled with TdT reaction mix for $60 \mathrm{~min}$ at $37^{\circ} \mathrm{C}$ in a dark humidified chamber. The reaction was stopped with $2 \mathrm{X}$ SSC solution, followed by
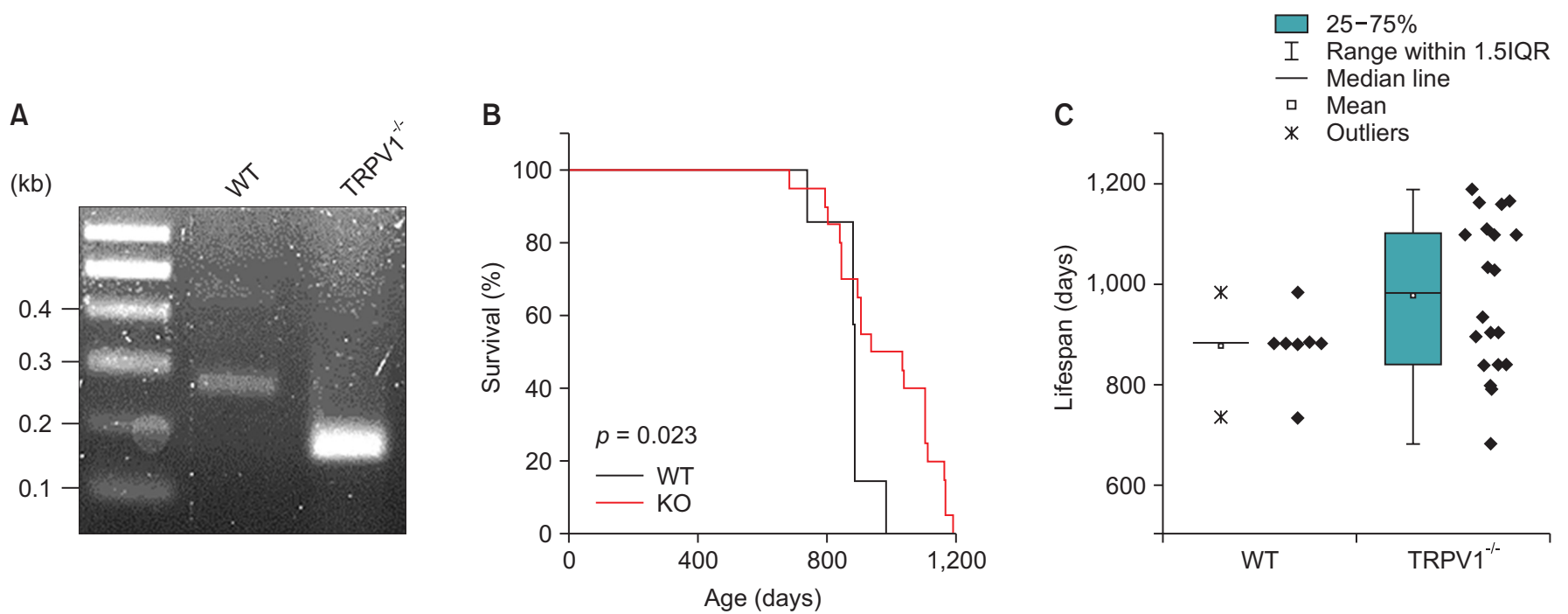

Fig. 1. Prolonged lifespan of mice lacking TRPV1 gene (A) PCR products obtained from genotyped WT and TRPV1 KO mice were 289 bp and 176 bp, respectively. (B) Kaplan-Meyer survival curves for male WT and TRPV1 KO mice are shown. (C) Box plot showing the survival data. WT and TRPV1 ${ }^{-1-}$ represent wild-type and TRPV1 $\mathrm{KO}$, respectively. 
washing three time in PBS. Counter staining was carried out by incubating with $5 \mu \mathrm{g} / \mathrm{mL}$ PI for $10 \mathrm{~min}$ at room temperature in the dark. TUNEL-positive cells were observed using a confocal laser scanning microscope (Olympus).

\section{Statistical analysis}

The data are represented as the mean \pm S.D. Significant differences between groups were evaluated using paired Student's $t$-test and the log-rank test for lifespan (OriginPro 8, Northampton, MA, USA). A value of $p<0.05$ was considered to be significant.

\section{RESULTS}

\section{Long-lived TRPV1 KO mice}

To determine whether TRPV1 is associated with longevity, WT and TRPV1 KO mice were housed until death in a pathogen-free animal area. TRPV1 genotypes were analyzed using specific primers with sequences provided by Jackson Laboratory. Male mice identified as homozygous TRPV1 KO were used in this study. Homozygous TRPV1 KO and WT mice yielded product sizes of $176 \mathrm{bp}$ and 289 bp, respectively (Fig. 1A). TRPV1 KO mice were significantly longer lived, which is in line with a previous study, as shown by their survival curves compared to WT mice ( $p$ $=0.023$, Fig. 1B). The lifespan was increased by $11.8 \%$ in male TRPV1 KO mice compared to WT mice. TRPV1 KO males lived approximately 100 days longer than WT mice, on average, and the maximum lifespan was markedly extended in TRPV1 KO mice (Fig. 1C). The TRPV1 KO mice were developmentally normal and fertile.

\section{Expression of TRPV1 in mouse testis}

The levels of TRPV1 mRNA and protein expression in the testes were determined by RT-PCR and IHC, respectively. Protein expression levels were compared between young (seven-week-old) and aged (32-month-old) mice. The testes expressed TRPV1 mRNA (Fig. 2A). Immunohistochemistry data showed that the expression of TRPV1 protein was increased in testes of older mice compared to young mice. TRPV1 was predominantly expressed in the Leydig cells of young mice, but a different expression pattern appeared in old testes. TRPV1 was expressed in the entire region of the older mice testes, and its expression was localized to the perinucleus area and the plasma membrane of testicular cells, including germ, Sertoli, and Leydig cells (Fig. 2B). As shown in Fig. 2C, the levels of TRPV1 protein expression were increased by approximately three-fold in testes obtained from aged mice compared to young mice.
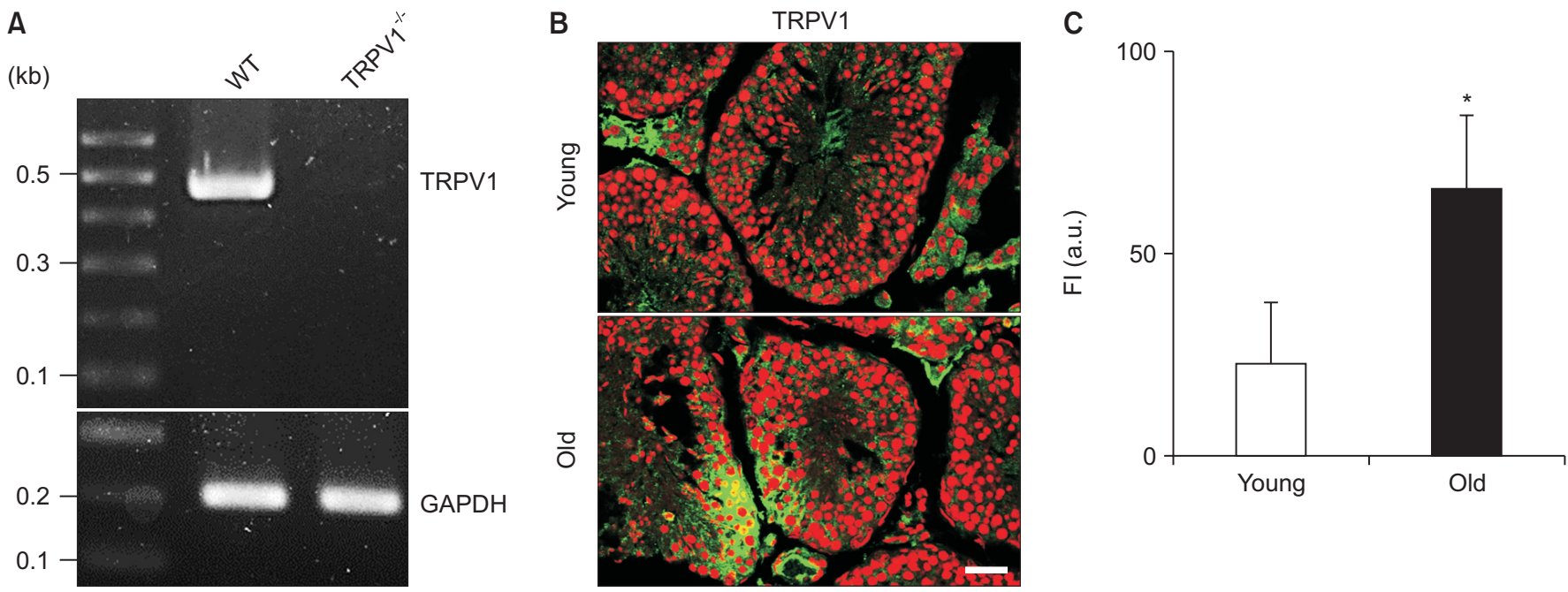

Fig. 2. Increased TRPV1 expression in old mice testes. (A) RT-PCR products for TRPV1 in testes. GAPDH was used as a loading control. (B) TRPV1 immunostaining in testes of young and old mice. Green and red indicate FITC fluorescence and PI, respectively. Scale bar, $30 \mu \mathrm{m}$. (C) Normalized fluorescence intensity of TRPV1 protein expression in testes of young and old mice. The fluorescence intensity was normalized to that of each background fluorescence intensity. ${ }^{*} p<0.05$ compared to the young mice. $\mathrm{Fl}$ and au represent fluorescence intensity and arbitrary unit, respectively. 
A

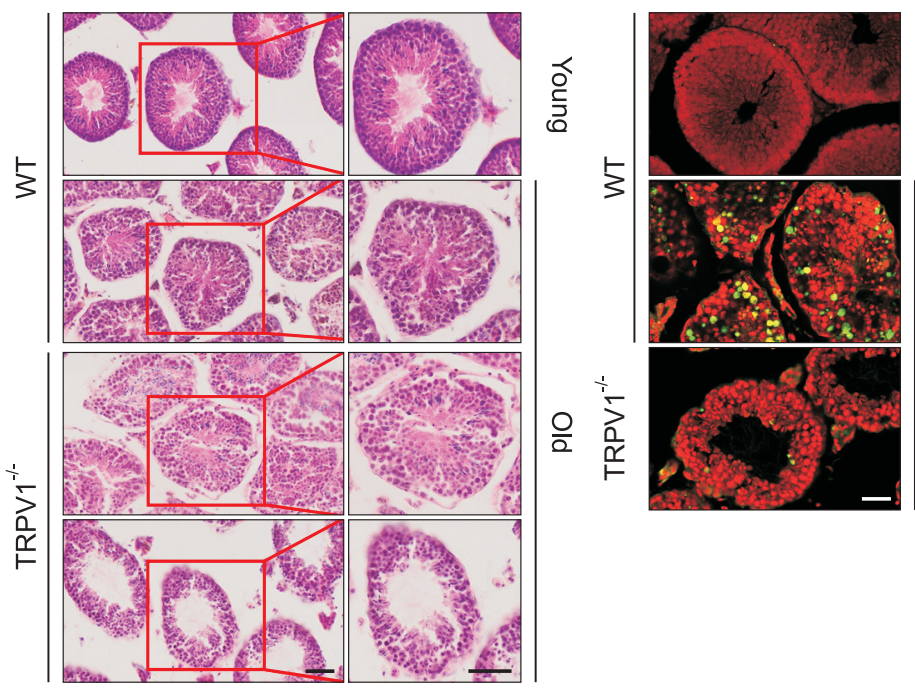

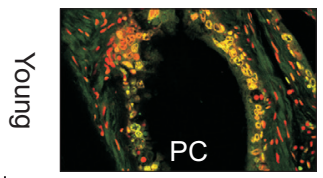

C

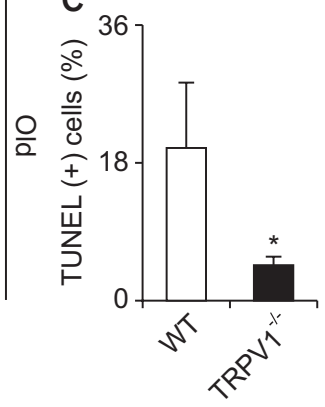

Fig. 3. Reduction of apoptotic cells in testes of TRPV1 KO mice. (A) Microphotographs of testis sections of young and old mice. The right panel shows enlarged images. Scale bar, $50 \mu \mathrm{m}$. (B) TUNEL-labeled apoptotic cells in the testicular cells. Scale bar, $30 \mu \mathrm{m}$. (C) Quantitative analysis of TUNEL-positive cells. The number of cells showing red (PI-stained cells) was divided by the number showing green (TUNEL-positive cells). ${ }^{*} p<0.05$ compared to the WT. Each bar represents the mean \pm SD of four experiments. PC and TUNEL (+) represent positive control treated with DNase I and TUNEL-positive, respectively.

\section{Reduction of apoptosis in testicular cells obtained from TRPV1 KO mice}

H\&E staining showed that spermatogenic cells were orderly arranged, and no pathological damage was observed in the seminiferous tubules of young mice. However, older mice showed a decrease in the number of testicular cells in the lumen of the seminiferous tubules compared to young mice (Fig. 3A). There were no histological changes between old WT and TRPV1 KO mice. Apoptotic cells determined by TUNEL assay were abundant in the old WT testes (Fig. 3B). Apoptotic cells were mainly found in the spermatocytes and Sertoli cells of old WT mice, whereas DNase-treated apoptosis (positive control, PC) was higher in the seminiferous epithelium. Quantitative analysis showed a significant reduction in old TRPV1 KO compared to WT mice (Fig. 3C, $p<0.01$ ).

\section{DISCUSSION}

Our study showed a significant increase in lifespan when lacking the TRPV1 gene. TRPV1 KO male mice lived $12 \%$ longer than their WT counterparts. The lifespan and longevity of TRPV1 KO mice examined in our study were similar to the findings of Riera et al. (2014), who demonstrated that TRPV1 KO mice showed a youthful metabolic profile when they are older compared to WT mice of the same age. The KO mice exhibited improvements in special memory and motor coordination skills that decreased with age. This report suggests that TRPV1 KO mice may have a slower progression of aging compared to WT. Currently, we do not know exactly how TRPV1 regulates aging and lifespan. It is hard to believe that a single gene regulates lifespan. It seems likely that TRPV1 controls many other molecules that affect cell proliferation and apoptosis in the body. TRPV1 is expressed in the testes (Barnes et al., 1998; Mizrak and van Dissel-Emiliani, 2008; De Toni et al., 2016), and TRPV1 activation induces apoptosis of many types of cells (Ramirez-Barrantes et al., 2016; Hou et al., 2019). Our study focused on testicular aging of TRPV1 KO mice by investigating cytological and histological changes.

Various testicular changes occur in old mice, including the reduction of testicular volume (Gosden et al., 1982; Wolf et al., 2000), accumulation of lipofuscin (age pigment) in Leydig cells (Miquel et al., 1978), multiple alterations in the Sertoli cell population (Cummins et al., 1994), loss of germ cells, and molecular alterations (Santiago et al., 2019). Our study showed decreases in the diameter and volume of seminiferous tubules and the number of Sertoli and germ cells in old mice. However, there was no changes in the number of Leydig cells between young and older mice. The expression of TRPV1 increased in the testes of older mice. Older mice showed an increased number of apoptotic cells in the seminiferous tubules compared to young mice. Interestingly, there were no significant differences in the number of testicular cells between old WT and TRPV1 KO mice. However, old TRPV1 KO mice showed lower apoptosis in the testes than WT mice. Therefore, TRPV1 was identified as a molecule that is changed in the testes of old mice. It is thought that 
other factors such as low androgen levels, mitochondrial dysfunction, high oxidative stress and inflammatory factors may still contribute to testicular degeneration in TRPV1 KO mice.

What does it mean to reduce apoptosis in the testes of TRPV1 KO mice? Apoptosis is known to be a major cause of germ cell loss (Gunes et al., 2016) and aging (Shen and Tower, 2009). Barnes et al. (1998) reported that the percentage of seminiferous tubules with apoptotic cells was not significantly altered with age (Barnes et al., 1998). Jara et al. (2004) proved that not all organs in mice have the same response to aging. Apoptosis occurred in the epididymis and ventral prostate, but the seminal vesicles and other prostatic lobes were not affected (Jara et al., 2004). The role of apoptosis is controversial in aging. Apoptosis protects tissues by removing inappropriate and damaged cells when cells are exposed to stressful environments. However, constant stress, such as aging, can increase apoptotic resistance, resulting in the improper survival of cells that cannot function normally (Pollack and Leeuwenburgh, 2001). Aging is associated with decreased apoptosis in several cell types (Tower, 2015). On the other hand, aging increases apoptotic signaling and the testicular apoptosis that is responsible for the decline of testicular function (Jara et al., 2004; Yang et al., 2015). Based on our results, we believe that apoptosis is associated with testicular aging, and high expression levels of TRPV1 in old mice induce testicular apoptosis. TRPV1 may not be directly related to the loss of testicular cells.

A limitation of this study is that specific markers to identify Sertoli, Leydig, and germ cells were not applied. Further study is needed to investigate the associations between TRPV1, testosterone, and Leydig and Sertoli cells. In addition, the role of TRPV1 in testicular aging should be identified in more detail. In conclusion, we confirmed that TRPV1 is associated with testicular apoptosis. In testes, TRPV1 expression levels were highly increased in old mice. In addition, TRPV1 KO old mice showed a longer lifespan and a lower level of apoptosis in the testes compared with those in WT mice. Our results suggest that TRPV1 may be associated with testicular aging.

\section{CONFLICTS OF INTEREST}

No potential conflict of interest relevant to this article was reported.

\section{ACKNOWLEDGEMENTS}

This work was supported by the National Research Foundation of Korea (NRF) grant funded by the Korea government (MSIT, NRF-2018R1A2B6001446 and NRF2015R1A-5A2-008833). Professor Seog Bae OH (Seoul National University, Seoul, South Korea) kindly provided TRPV1 KO mice, which were purchased from Jackson Laboratory, to professor Dong Kun Lee (Gyeongsang National University.

\section{AUTHOR'S AFFILIATION, POSITION AND ORCID NO.}

AS Siregar, Gyeongsang Nat'l Univ., Ph.D Student, https://orcid.org/0000-0001-7069-4202

MM Nyiramana, Gyeongsang Nat'l Univ., Ph.D Student, https://orcid.org/0000-0002-0018-8934

EJ Kim, Gyeongsang Nat'l Univ., Research Fellow, https://orcid.org/0000-0003-1068-9334

EJ Shin, Gyeongsang Nat'1 Univ., M.S. Student, https://orcid.org/0000-0002-5673-5079

CW Kim, Sungkyunkwan Univ., Professor, https://orcid.org/0000-0002-6663-0764

DK Lee, Gyeongsang Nat'l Univ., Professor, https://orcid.org/0000-0001-9969-0117

SG Hong, Gyeongsang Nat'l Univ., Professor, https://orcid.org/0000-0002-2117-9324

J Han, Gyeongsang Nat'l Univ., Professor, https://orcid.org/0000-0002-3523-008X

D Kang, Gyeongsang Nat'l Univ., Professor, https://orcid.org/0000-0001-7402-7298

\section{REFERENCES}

Barnes CJ, Covington BWt, Cameron IL, Lee M. 1998. Effect of aging on spontaneous and induced mouse testicular germ cell apoptosis. Aging (Milano). 10:497-501.

Caterina MJ, Leffler A, Malmberg AB, Martin WJ, Trafton J, Petersen-Zeitz KR, Koltzenburg M, Basbaum AI, Julius D. 2000. Impaired nociception and pain sensation in mice lacking the capsaicin receptor. Science. 288:306-313.

Caterina MJ, Schumacher MA, Tominaga M, Rosen TA, Levine JD, Julius D. 1997. The capsaicin receptor: a heat-activated ion channel in the pain pathway. Nature. 389:816-824.

Cummins JM, Jequier AM, Kan R. 1994. Molecular biology of human male infertility: links with aging, mitochondrial genetics, and oxidative stress? Mol Reprod Dev. 37:345-362. 
De Toni L, Garolla A, Menegazzo M, Magagna S, Di Nisio A, Sabovic I, Rocca MS, Scattolini V, Filippi A, Foresta C. 2016. Heat Sensing Receptor TRPV1 Is a Mediator of Thermotaxis in Human Spermatozoa. PLoS One. 11:e0167622.

Gosden RG, Richardson DW, Brown N, Davidson DW. 1982. Structure and gametogenic potential of seminiferous tubules in ageing mice. J Reprod Fertil. 64:127-133.

Gunes S, Hekim GN, Arslan MA, Asci R. 2016. Effects of aging on the male reproductive system. J Assist Reprod Genet. 33: 441-454.

Handelsman DJ. 2006. Testosterone: use, misuse and abuse. Med J Aust. 185:436-439.

Hou N, He X, Yang Y, Fu J, Zhang W, Guo Z, Hu Y, Liang L, Xie W, Xiong H, Wang K, Pang M. 2019. TRPV1 Induced Apoptosis of Colorectal Cancer Cells by Activating Calcineurin-NFAT2p53 Signaling Pathway. Biomed Res Int. 2019:6712536.

Jara M, Carballada R, Esponda P. 2004. Age-induced apoptosis in the male genital tract of the mouse. Reproduction. 127:359-366.

Martins D, Tavares I, Morgado C. 2014. "Hotheaded": the role OF TRPV1 in brain functions. Neuropharmacology. 85:151157.

Miquel J, Lundgren PR, Johnson JE, Jr. 1978. Spectrophotofluorometric and electron microscopic study of lipofuscin accumulation in the testis of aging mice. J Gerontol. 33:3-19.

Mizrak SC, Gadella BM, Erdost H, Ozer A, van Pelt AM, van Dissel-Emiliani FM. 2008. Spermatogonial stem cell sensitivity to capsaicin: an in vitro study. Reprod Biol Endocrinol. 6:52.

Mizrak SC, van Dissel-Emiliani FM. 2008. Transient receptor potential vanilloid receptor-1 confers heat resistance to male germ cells. Fertil Steril. 90:1290-1293.

Muradian KK, Utko NA, Fraifeld V, Mozzhukhina TG, Pishel IN,
Litoshenko AY. 2002. Superoxide dismutase, catalase and glutathione peroxidase activities in the liver of young and old mice: linear regression and correlation. Arch Gerontol Geriatr. 35:205-214.

Pollack M, Leeuwenburgh C. 2001. Apoptosis and aging: role of the mitochondria. J Gerontol A Biol Sci Med Sci. 56:B475482.

Ramirez-Barrantes R, Cordova C, Poblete H, Munoz P, Marchant I, Wianny F, Olivero P. 2016. Perspectives of TRPV1 Function on the Neurogenesis and Neural Plasticity. Neural Plast. 2016:1568145.

Riera CE, Huising MO, Follett P, Leblanc M, Halloran J, Van Andel R, de Magalhaes Filho CD, Merkwirth C, Dillin A. 2014. TRPV1 pain receptors regulate longevity and metabolism by neuropeptide signaling. Cell. 157:1023-1036.

Santiago J, Silva JV, Alves MG, Oliveira PF, Fardilha M. 2019. Testicular Aging: An Overview of Ultrastructural, Cellular, and Molecular Alterations. J Gerontol A Biol Sci Med Sci. 74:860-871.

Shen J, Tower J. 2009. Programmed cell death and apoptosis in aging and life span regulation. Discov Med. 8:223-226.

Tenover JS. 2003. Declining testicular function in aging men. Int J Impot Res. 15 Suppl 4:S3-8.

Tower J. 2015. Programmed cell death in aging. Ageing Res Rev. 23:90-100.

Wolf KN, Wildt DE, Vargas A, Marinari PE, Kreeger JS, Ottinger MA, Howard JG. 2000. Age-dependent changes in sperm production, semen quality, and testicular volume in the black-footed ferret (Mustela nigripes). Biol Reprod. 63:179187.

Yang J, Zong X, Wu G, Lin S, Feng Y, Hu J. 2015. Taurine increases testicular function in aged rats by inhibiting oxidative stress and apoptosis. Amino Acids. 47:1549-1558. 